\title{
Queixas álgicas e distúrbios musculoesqueléticos em profissionais da enfermagem:
}

\section{uma revisão integrativa}

\author{
Pain complaints and musculoskeletal disorders in nursing professionals: an integrative review \\ Quejas de dolor y trastornos musculoesqueléticos en profesionales de enfermería: una revisión \\ integradora
}

Recebido: 06/11/2021 | Revisado: 12/11/2021 | Aceito: 13/11/2021 | Publicado: 22/11/2021

\author{
Nezilda Ramos da Silva Malcher \\ ORCID: https://orcid.org/0000-0002-7985-5356 \\ Centro Universitário da Amazônia, Brasil \\ E-mail: nezildaramos.malcher@gmail.com \\ Antônio Augusto da Cunha Palheta \\ ORCID: https://orcid.org/0000-0002-0262-9537 \\ Centro Universitário da Amazônia, Brasil \\ E-mail: augustocunha1979@gmail.com \\ Eliane Ferreira Marinho \\ ORCID: https://orcid.org/0000-0002-3323-6293 \\ Centro Universitário da Amazônia, Brasil \\ E-mail: elianefmarinho@yahoo.com.br
}

\begin{abstract}
Resumo
Objetivo: Investigar as principais queixas álgicas indicativas de Distúrbio Osteomuscular Relacionado ao trabalho (DORT) entre profissionais da enfermagem. Metodologia: Trata-se de uma revisão integrativa da literatura, com pesquisa nas bases de dados: Literatura Latino-Americana e do Caribe em Ciências da Saúde (LILACS), Scientific Electronic Library Online (SciELO), Medical Literature Analysis and Retrieval System Online (MEDLINE), National Library of Medice (PubMed) e Google Acadêmico. A partir dos descritores, seguindo os critérios de inclusão e exclusão, reunindo preferencialmente, artigos publicados nos últimos 5 anos, na língua portuguesa e inglesa, que apresentassem em seu conteúdo relação direta com o tema proposto. Resultados e discussão: Selecionou-se 11 artigos. As principais queixas e sintomatologias indicativas de DORT envolvem, principalmente, quadros álgicos em região lombar, cervical, membros superiores e inferiores, sendo a lombalgia o principal sintoma auto referido pelos profissionais da enfermagem. Os principais fatores etiológicos dos DORTS relacionam-se às posturas exercidas durante o labor, às longas jornadas de trabalho e aos altos índices de exposição mecânica, além do levantamento e manuseio de materiais que resultam em altas cargas nas regiões do corpo mais relatadas. Conclusão: Existe um elevado índice de dores relacionadas ao trabalho nos profissionais de enfermagem. As principais queixas são de quadros álgicos prevalentes na região lombar, associados à organização do trabalho e à biomecânica exercida durante o labor. Sugere-se a realização de estudos mais aprofundados acerca da temática, que possam embasar intervenções cada vez mais assertivas em função da qualidade de vida no trabalho e da promoção da saúde dos profissionais.
\end{abstract}

Palavras-chave: Distúrbio osteomuscular relacionado ao trabalho; Lesão por esforço repetitivo; Dor osteomuscular; Enfermeiros.

\begin{abstract}
Objective: To investigate the main pain complaints indicative of Work-Related Musculoskeletal Disorder (WMSD) among nursing professionals. Methodology: This is an integrative literature review, with a search in the following databases: Latin American and Caribbean Health Science Literature (LILACS), Scientific Electronic Library Online (SciELO), Medical Literature Analysis and Retrieval System Online (MEDLINE), National Library of Medice (PubMed) and Google Academic. Articles published in the last 5 years (2016 - 2021), in Portuguese and English, that presented in their direct content with the proposed theme were used. Results and discussion: 11 articles were selected. The main complaints and symptoms indicative of WMSD mainly involve pain in the lumbar region, neck, upper and lower limbs, with low back pain or the main symptom self-reported by nursing professionals. The main etiological factors of WMSD are related to postures exercised during work, long working hours and high rates of mechanical exposure, in addition to the lifting and handling of materials that result in high loads in the most reported regions of the body. Conclusion: There is a high rate of work-related pain in nursing professionals. The main complaints are pain conditions with prevalence in the lumbar region, associated with the organization of work and the biomechanics exercised during work activities. It is suggested to carry out more in-depth studies on the subject, which can support increasingly assertive interventions in terms of quality of life at work and health promotion for professionals.
\end{abstract}


Keywords: Work-related musculoskeletal disorder; Repetitive strain injury; Musculoskeletal pain; Nurses.

\section{Resumen}

Objetivo: Investigar las principales quejas de dolor indicativas de trastorno musculoesquelético relacionado con el trabajo (WMSD) entre los profesionales de enfermería. Metodologia: Se trata de una revisión de literatura integradora, con búsqueda en las siguientes bases de datos: Literatura Latinoamericana y del Caribe en Ciencias de la Salud (LILACS), Scientific Electronic Library Online (SciELO), Medical Literature Analysis and Retrieval System Online (MEDLINE), National Library of Medice (PubMed) y Google académico. Se utilizaron artículos publicados en los últimos 5 años (2016 - 2021), en portugués e inglés, que presentaban en su contenido una relación directa con la temática propuesta. Resultados y discusión: se seleccionaron 11 artículos. Las principales quejas y síntomas indicativos de WMSD involucran principalmente dolor en las extremidades lumbares, cervicales, superiores e inferiores, siendo la lumbalgia el principal síntoma autoinformado por los profesionales de enfermería. Los principales factores etiológicos de DORTS están relacionados con las posturas ejercidas durante el trabajo, las largas jornadas laborales y los altos índices de exposición mecánica, además del levantamiento y manipulación de materiales que resultan en elevadas cargas en las regiones del cuerpo más reportadas. Conclusión: Existe una alta tasa de dolor relacionado con el trabajo en los profesionales de enfermería. Las principales quejas son las condiciones de dolor con predominio en la región lumbar, asociadas a la organización del trabajo y la biomecánica ejercida durante las actividades laborales. Se sugiere realizar estudios más profundos sobre el tema, que puedan sustentar intervenciones cada vez más asertivas en términos de calidad de vida en el trabajo y promoción de la salud de los profesionales.

Palabras clave: Trastorno musculoesquelético relacionado con el trabajo; Lesión por esfuerzo repetitivo; Dolor musculoesquelético; Enfermeras.

\section{Introdução}

Muitos trabalhadores, quando submetidos à intensas e longas jornadas de trabalho, apresentam com o tempo diversos e distintos distúrbios musculoesqueléticos (Lucca, 2017). A cada dia que passa, percebe-se que há necessidade de aprimorar conhecimentos que possam dar suporte a cada classe trabalhadora a ultrapassar as dificuldades que advém de tais distúrbios. Ao conjunto de sintomas adivindos de distúrbios musculoesqueléticos, foi dado o termo LER- Lesões por esforços repetitivos, e posteriormente, DORT- Distúrbio Osteomuscular Relacionado ao Trabalho. O termo DORT é o mais abrangente, que se refere aos distúrbios ou doenças do sistema musculoesquelético, relacionados, comprovadamente ou não, ao trabalho e se mostram com grande evidência dentro do ambiente de trabalho, que podem acometer muitos trabalhadores de distintas ocupações (Ferri \& Xavier, 2015).

Os DORT são um grupo de lesões que constituem doenças ocupacionais, que têm repercussões sociais, bem como psicológicas e se caracterizam por sintomas dolorosos que acometem tendões, músculos, nervos, ligamentos e outras estruturas responsáveis pelos movimentos (Ferri \& Xavier, 2015; Almeida et al., 2016; Martins \& Correa, 2017). Essas doenças podem ser doenças inflamatórias e degenerativas que afetam os músculos, tendões, ligamentos, articulações, cartilagem, nervos periféricos, vasos sanguíneos de suporte ou discos espinhais (Anap et al., 2013; Punnett \& Wegman, 2004).

Esses distúrbios são causados por fatores como as condições precárias de trabalho, repetitividade, má postura, resistência pessoal, esforço, velocidade no cumprimento da atividade, estado psicossocial do indivíduo, bem como outros fatores externos e internos que influenciam a sua etiologia como a iluminação, ruídos, frio, vibração, umidade, espaço físico inadequado. As disfunções podem ser adquiridas por múltiplas causas, podendo surgir devido um conjunto de fatores no ambiente de trabalho, como a repetitividade do movimento ou manutenção de posturas inadequadas, na maioria das vezes, atingindo inclusive, dimensões biopsicossociais (Costa \& Dias, 2015; Santos \& Andrade, 2011).

Em enfermeiros, o principal fator de risco à etiologia das DORT é o manuseio dos pacientes, devido às posições extremas adotadas durante a prestação destes cuidados (Fonseca \& Serranheira, 2006). No entanto, é necessário dar atenção não só as atividades ligadas a manejo e transferência de pacientes, mas também as demais atividades de trabalho que requerem movimentos repetitivos e precisos com altas exigências de atenção nos procedimentos realizados pelos enfermeiros no ambiente de trabalho (Sousa et al., 2015). 
Os profissionais de saúde são uma das maiores categorias de trabalhadores do mundo e por se dedicarem ao cuidado devem ser valorizadas (Neves \& Serranheira, 2014). No ambiente hospitalar, a equipe de enfermagem permanece $24 \mathrm{~h}$ atendendo e dando continuidade ao trabalho de assistência nas unidades de internação. Assim, essa classe profissional é a que mais se relaciona com o paciente, já que durante a maior parte do tempo há um profissional de saúde ao lado do leito (Vieira \& Alcântara, 2013). Diante de suas atividades laborais, este é um grupo altamente vulnerável a desenvolver problemas relacionados à saúde física e mental, pela natureza do trabalho executado, incluindo situações desgastantes que enfrentam em suas atividades diárias, como a alta demanda de serviços e múltiplos fatores psicossociais e de transferência que recebem de seus pacientes (Freitas, 2012).

O desgaste físico pode levar a uma série de manifestações. A exaustão física nos profissionais da saúde é comum, pois no seu dia a dia realizam atividades que demandam posturas corporais extremas da coluna vertebral, por exemplo, muitas horas de pé, em posições desconfortáveis e, em alguns casos, a falta de pessoal faz com que outros profissionais sejam sobrecarregados com o trabalho. São diversas situações que os expõem a fatores de risco profissionais, como necessidades biomecânicas e fisiológicas que vão além de suas capacidades funcionais. No caso da equipe de enfermagem, além dos cuidados contínuos, diretamente relacionados aos usuários que necessitam, são realizadas diversas tarefas administrativas, tornando, nesse sentido, o ambiente de trabalho, um local altamente estressante para esses profissionais (Shieh et al., 2016; Vieira \& Alcântara, 2013).

Os DORT na enfermagem derivam de tarefas fisicamente exigentes, como ajudar os pacientes à ajustar a posição, mantendo uma inclinação para a frente, postura torcida e levantar pacientes pesados (Cilliers \& Maart, 2013; Jellad et al., 2013). Para o seu diagnóstico, realiza-se a análise clínica em consonância com exames de imagem (Punnett \& Wegman, 2004). Podendo ser tratada após o diagnóstico preciso, pois DORT é composto por um conjunto de doenças que se não forem bem diagnosticadas, o tratamento não será eficaz (Reis, 2016). Sua terapêutica abrange não só procedimentos cirúrgicos, mas também infiltrações, órteses, fisioterapia, medicamentos como os anti-inflamatórios e os analgésicos (Pinhatti et al. 2018).

Os profissionais de enfermagem, que desenvolvem suas atividades em diversos locais, dentre os quais encontram-se os ambulatórios e as instituições hospitalares, realizam atividades de forma contínua (Chung et al. 2013), que exigem constante atenção, esforço físico, posições inadequadas, movimentos repetitivos, excessiva jornada de trabalho, tornando-os expostos a diversos fatores de riscos ocupacionais e a um nível elevado de predisposição ao risco de adoecimento no trabalho (Lelis et al., 2012).

Assim, os DORTs compõem a principal causa de lesões relacionadas ao trabalho entre os enfermeiros (Chung et al., 2013) e estão associados com dor e alterações musculoesqueléticas, que são conceituados por muitos estudiosos como um problema de saúde pública que afeta os enfermeiros, causando o afastamento temporário ou até mesmo definitivo, consequentemente gerando um custo alto com tratamentos, substituições ou até mesmo indenizações (Sorour \& El-Maksoud, 2012; Sousa et al., 2015; Paula \& Amaral, 2019).

Para a Organização Internacional do Trabalho (OIT) e a Organização Mundial da Saúde (OMS) os DORT são considerados como uma "nova epidemia" que deve ser investigada e resolvida (Luan et al., 2018). É importante compreender que os profissionais da enfermagem no Brasil fazem parte de uma profissão caracterizada por ter turnos rotativos de trabalho contrários ao ritmo biológico do organismo, associado às longas jornadas, frequentemente justificada por falta de pessoal, o que culmina com um ritmo exaustivo de trabalho, em regime de plantões, carga horária elevada, trabalho noturno e desenvolvimento de tarefas que demandam sobrecarga horária laboral, podem deixar os enfermeiros suscetíveis a contrair o DORT (França et al. 2012; Lucca \& Rodrigues, 2015; Bezerra \& Costa, 2016).

Nessa perspectiva, a presente pesquisa tem intuito de levantar as principais queixas álgicas indicativas de DORT, entre profissionais da enfermagem, através de uma revisão integartiva da bibliografia. Identificando as principais etiologias e os sintomas adquiridos pelos trabalhadores de enfermagem, sendo de grande relevância, pois irá contribuir para contínua discussão 
e investigação acerca do tema, reunindo resultados relevantes do acervo literário, na intenção de contribuir para que os índices de casos de DORT possam ser minimizados da melhor forma possível.

\section{Metodologia}

O presente estudo é caracterizado como uma revisão de literatura do tipo integrativa, com abordagem qualitativa (Koche, 2011; Estrela, 2018). O estudo foi realizado por meio de levantamento bibliográfico, em seis fases do processo de produção:

$1^{\circ}$ ) Elaboração da pergunta norteadora, com definição dos descritores utilizados, que são Disturbio osteomuscular relacionado ao trabalho, Lesão por esforço repetivivo, Dor osteomuscular, Enfermeiros e seus correspondentes na língua inglesa: Work-related musculoskeletal disorder, Repetitive strain injury, Musculoskeletal pain, Nurses.

$2^{\circ}$ ) Busca ampla na literature, diversificada em base de dados eletrônicas, a saber, Literatura Latino-Americana e do Caribe em Ciências da Saúde (LILACS), Scientific Electronic Library Online (SciELO), Medical Literature Analysis and Retrieval System Online (MEDLINE), National Library of Medice (PubMed), e Google Acadêmico.

$3^{\circ}$ ) Coleta de dados com a utilização de um instrumento previamente elaborado, organizando, registrando e assegurando que a totalidade dos dados relevantes sejam extraídos.

$4^{\circ}$ ) Análise crítica dos estudos incluídos, ponderando com rigor, as características de cada estudo.

$5^{\circ}$ ) Discussão a partir da interpretação e síntese dos resultados, comparando dados.

$6^{\circ}$ ) Apresentação da revisão integrativa, após resumo, simplificação, e organização dos dados extraídos das fontes primárias. Após redução, exposição e comparações, para elaboração da conclusão e revisão completa de forma clara, no artigo final.

Como critérios de inclusão, foram utilizados artigos publicados em revistas indexadas, abordando o tema, pesquisados a partir dos descritores, preferencialemente nos últimos 5 anos, redigidos na língua portuguesa e inglesa que apresentem em seu conteúdo relação direta com o objetivo da corrente pesquisa. Foram excluídos, artigos que não fizeram jus aos critérios de inclusão, estudos de revisão de literatura, estudos de casos, estudos em andamento, superiores a cinco anos, e que não estabeleceram relevância com o tema proposto.

As buscas foram realizadas no período compreendido entre agosto a setembro de 2021.

\section{Resultados e Discussão}

Foram encontrados 805 artigos nas bases de dados consultadas. Após aplicação dos critérios de inclusão, exclusão e triagem, foram selecionados 11 artigos para compor a presente revisão integrativa. Todos avaliam a prevalência de DORT em profissionais da enfermagem. As características dos estudos encontrados podem ser observadas no Quadro 1 a seguir. 
Quadro 1 - Síntese dos principais achados sobre queixas álgicas e distúrbios musculoesqueléticos em profissionais da enfermagem.

\begin{tabular}{|c|c|}
\hline Autores (Ano) & Principais achados \\
\hline $\begin{array}{l}\text { Mazalo et al., } \\
(2021)\end{array}$ & $\begin{array}{l}\text { Estudo transversal, com } 25 \text { enfermeiros através da coleta de dados com o questionário } \\
\text { sociodemográfico e Questionário Nórdico de Sintomas Osteomusculares. Os enfermeiros entrevistados } \\
\text { apresentaram casos sugestivos de DORT. }\end{array}$ \\
\hline $\begin{array}{l}\text { Cargnin et } \\
\text { al., (2019) }\end{array}$ & $\begin{array}{l}\text { Estudo transversal, com } 301 \text { profissionais da enfermagem com aplicação do Questionário Nórdico de } \\
\text { Sintomas Osteomusculares e a Escala de Avaliação do Contexto de Trabalho. Houve associação entre } \\
\text { o trabalho e a lombalgia. }\end{array}$ \\
\hline $\begin{array}{l}\text { Elsherbeny et } \\
\text { al., (2018) }\end{array}$ & $\begin{array}{l}\text { Estudo transversal com } 311 \text { enfermeiras através de questionário sociodemográfico e Questionário } \\
\text { Nórdico de Sintomas Osteomusculares. Houve alta prevalencia de queixas musculoesqueléticas entre } \\
\text { as enfermeiras. }\end{array}$ \\
\hline $\begin{array}{l}\text { Brien et al., } \\
(2018)\end{array}$ & $\begin{array}{l}\text { Estudo quantitativo, descritivo, transversal com } 59 \text { enfermeiros através da aplicação do questionário } \\
\text { sociodemográfico e Questionário Nórdico de Sintomas Osteomusculares. Houve alta prevalência de } \\
\text { DORT entre os profissionais. }\end{array}$ \\
\hline $\begin{array}{l}\text { Pereira et } \\
\text { al., (2018) }\end{array}$ & $\begin{array}{l}\text { Estudo transversal com } 21 \text { profissionais da enfermagem, através da aplicação de um questionário com } \\
\text { questões fechadas e abertas sobre o tema. O diagnóstico de DORT está presente entre os profissionais. }\end{array}$ \\
\hline $\begin{array}{l}\text { Luan et al., } \\
(2018)\end{array}$ & $\begin{array}{l}\text { Estudo transversal com } 1179 \text { enfermeiros usando o Questionário Nórdico Padronizado. Foi encontrado } \\
\text { DORT em grande parte da amostra e uma parte considerável dela afirmou que os sintomas de limitam } \\
\text { seu trabalho }\end{array}$ \\
\hline $\begin{array}{l}\text { Chiwaridz et al., } \\
(2018)\end{array}$ & $\begin{array}{l}\text { Estudo transversal com } 117 \text { enfermeiros para analisar a prevalência, consequências e fatores associados } \\
\text { aos DORT, atraves do Questionário Nórdico de Sintomas Osteomusculares. O estudo constatou que os } \\
\text { DORTs são uma ocorrência comum entre os enfermeiros }\end{array}$ \\
\hline $\begin{array}{l}\text { Freire et al., } \\
(2017)\end{array}$ & $\begin{array}{l}\text { Estudo exploratório, descritivo, de abordagem quantitativa com } 20 \text { profissionais da enfermagem, } \\
\text { através da aplicação de questionário ergonômico. Houve grande incidência de lombalgia durante as } \\
\text { atividades laborais e após a jornada de trabalho. }\end{array}$ \\
\hline $\begin{array}{l}\text { Ribeiro et al., } \\
(2017)\end{array}$ & $\begin{array}{l}\text { Estudo transversal com } 409 \text { enfermeiros da atenção priária à saúde, através do Questionário Nórdico } \\
\text { de Sintomas Osteomusculares. Houve alta prevalência de sintomas de DORT, associados aos fatores } \\
\text { de risco na execução do labor. }\end{array}$ \\
\hline $\begin{array}{l}\text { Arvidsson et al., } \\
(2016)\end{array}$ & $\begin{array}{l}\text { Estudo observacional transversal com } 1591 \text { mulheres, destas, } 906 \text { eram enfermeiras de um Hospital } \\
\text { Universitário, através da aplicação do questionário sociodemográfico e Questionário Nórdico de } \\
\text { Sintomas Osteomusculares. Houve alta prevalência de DORT e incidência de dor lombar. }\end{array}$ \\
\hline $\begin{array}{l}\text { Rodrigues } \\
(2016)\end{array}$ & $\begin{array}{l}\text { Estudo descritivo, de âmbito quantitativo com } 34 \text { profissionais de enfermagem, através de um } \\
\text { questionário sociodemográfico, Questionário Nórdico de Sintomas Osteomusculares e de um censo de } \\
\text { ergonomia modificado. Foi identificada uma alta prevalência de DORT entre os profissionais. Os } \\
\text { aspectos organizacionais do trabalho foram contribuitivos para as ocorrências. }\end{array}$ \\
\hline
\end{tabular}

Legenda: DORT - Disturios Osteomusculaes Relacionados ao Trabalho.

Fonte: Autores.

De acordo com os achados alocados no quadro 1, entende-se que o nível físico de desgaste é um processo de perda lenta, porém considerável, de comprometimento e envolvimento pessoal nas tarefas executadas. Para alguns autores, a exaustão 
profissional é um conjunto de sinais e sintomas mal definidos, enquanto para outros é uma doença em si e um potencial problema de saúde pública (Rodrigues, 2016; Arvidson et al, 2016; Chiwaridz et al., 2018; Mazalo et al., 2021).

Elsherben et al., (2018) referem que a profissão de Enfermagem evidencia-se entre as ocupações estreitamente relacionadas com a prevalência de DORT e os destacam como o principal problema de saúde ocupacional nos enfermeiros.

Pereira et al., (2018) fizeram um estudo transversal com 21 profissionais da enfermagem do Hospital Municipal de JiParaná/PR, para Identificar a ocorrência de sinais e sintomas, principais queixas, regiões do corpo mais afetadas e identificar as atividades profissionais comprometidas pela DORT, através da aplicação de um questionário com questões fechadas e abertas sobre o tema. No que diz respeito ao tempo de serviço e a ocorrência do sintoma de dor associado a outros sintomas, o maior percentual ocorre no período 2 a 5 anos. A maior parte dos casos acomete trabalhadores da obstetrícia, pediatria e geriatria. $\mathrm{O}$ diagnóstico de DORT esteve presente em 30\% dos trabalhadores. A região do corpo mais afetada foi a dos membros superiores, prevalecendo os sintomas de dor e fadiga (Pereira et al., 2018)

Freire et al., (2017) também investigaram a ocorrência de lombalgia em 20 profissionais da enfermagem para identificar e correlacionar a incidência com a aplicabilidade dos princípios ergonômicos. A incidência de lombalgia durante as atividades foi de $80 \%$, e após a jornada de trabalho foi de $75 \%, 65 \%$ dos profissionais alegaram nunca terem recebido orientações ergonômicas, resultando na aplicabilidade insuficiente de tais princípios.

Em mais um estudo sobre a DORT em enfermeiros, realizado em Portugal por Ribeiro et al., (2017) com uma amostra $(n=409)$, composta principalmente pelo sexo feminino $(84,0 \%)$, para descrever os sintomas de DORT autorrelatados pelos enfermeiros, e caracterizando as principais tarefas laborais que podem apresentar fatores de risco para o seu desenvolvimento, observou-se que a região lombar foi a região corporal mais acometida (63,1\%), seguida pela cervical, dorsal, ombros e punho/mão. No mesmo período, o absenteísmo relacionado a essas queixas foi alto $(51,4 \%)$ e fortemente relacionado ao trabalho em pé (48,8\%), flexão de tronco (42,3\%), rotação de tronco (40,6\%), aplicação de força com as mãos ou dedos (37,3\%), trabalho sentado $(36,6 \%)$ e movimento repetitivo do braço $(34,3 \%)$. Este estudo mostrou que existe uma grande semelhança no que concerne a região mais afetada pelos sintomas. Houve associações entre a prevalência de sintomas de DORT em diferentes regiões do corpo e algumas características individuais. Notou-se que os enfermeiros estão expostos a fatores de risco, associados a uma alta prevalência de sintomas de DORT, como posturas antálgicas e extremas mantidas por períodos prolongados e sua repetitividade contribuirão para essa ocorrência (Ribeiro et al. 2017).

Outra evidência é o estudo realizado por Rodrigues (2016) sobre os DORT em 34 profissionais de enfermagem, para identificar sintomas musculoesqueléticos nos profissionais que atuam em uma Unidade de Terapia Intensiva de um Hospital Federal do Rio de Janeiro. Foi possível identificar uma prevalência de sintomas osteomusculares de 94,11\% entre os trabalhadores nos últimos 12 meses e de 58,82\% nos últimos 7 dias. As regiões corporais mais referidas foram a parte inferior das costas $(79,41 \%)$, pescoço $(64,70 \%)$ e parte superior das costas $(58,82 \%)$.

Mazalo et al., (2021) ao buscar conhecer os distúrbios osteomusculares relacionados ao trabalho, constataram que os enfermeiros entrevistados apresentaram casos sugestivos de DORT em 56\%, sendo que 85,7\% relataram que os sintomas pioravam com o exercício da profissão, onde as regiões anatômicas mais afetadas pelos sintomas foram a lombar $72 \%$, seguida pelos ombros e pés com $68 \%$, respectivamente

Brien et al., (2018) realizaram uma pesquisa com 59 enfermeiros de quatro unidades de reabilitação de pacientes com lesão medular na África do Sul para determinar a prevalência de DORT na enfermagem, as regiões corporais mais afetadas, os fatores de risco de trabalho percebidos e estratégias de enfrentamento na prevenção das doenças. Sendo que, 57,6\% experienciaram sintomatologia de DORT nos últimos 12 meses. As taxas de prevalência foram mais elevadas na região lombar (73,5\%), seguido de ombros $(41,2 \%)$, joelhos e tornozelos $(26,5 \%)$ e região torácica $(23,53 \%)$ e o fator de risco de trabalho mais comum percebido foi flexão e rotação de coluna de forma repetitiva. 
Luan et al., (2018) também investigaram a prevalência de DORT em 1179 enfermeiros de 15 hospitais distritais no Vietnan. Nesta pesquisa verificaram uma taxa de prevalência de sintomatologia de DORT em 74,7\% nos últimos 12 meses e $41,1 \%$ nos últimos sete dias, sendo que 37,8\% referiram que os sintomas limitavam o suas atividades laborativas. A sintomatologia registou-se mais comum na região lombar (44,4\%), região cervical $(44,1 \%)$, região dorsal $(32,7 \%)$ e ombros (29,7\%). Sendo que 37,8\% dos profissionais reclamaram que os sintomas de DORT limitavam seu trabalho. Os resultados mostraram que as mulheres eram 2,1 vezes mais propensas a desenvolver lesões musculoesqueléticas do que os homens, os enfermeiros com história prévia de DORT tinham maior tendência para desenvolver sintomatologia nos últimos 12 meses, bem como os que apresentavam sintomas de sofrimento psíquico.

Chiwaridzo et al., (2018) também realizaram um estudo num hospital com 117 enfermeiros, em Zimbabué para analisar a prevalência, consequências e fatores associados aos DORT e verificaram que 82,1\% dos enfermeiros apresentaram sintomas de DORT nos últimos 12 meses, sendo a região lombar a mais frequente $(67,9 \%)$. Os autores constataram associações significativas entre os DORT e o maior tempo de experiência profissional, menor qualificação e não ter formação sobre ergonomia. Os fatores etiológicos mais percepcionados para as queixas lombares foram os movimentos incorretos de flexão e rotação da coluna vertebral, longos períodos de pé na prestação de cuidados a grande número de pacientes, elevação, transferência de pacientes dependentes e pausas inadequadas.

Arvidsson et al., (2016) realizaram um estudo com 1591 mulheres em cinco grupos ocupacionais, destas, 906 profissionais da enfermagem de um Hospital Universitário da Estórnia. O intuito foi verificar a prevalência de DORT e explorar a sua associação com os fatores ocupacionais e pessoais. Os autores notaram a prevalência de DORT em 70\%, a incidência de lombalgia em 57\% e de cervicalgia em 56\% da amostra. As queixas álgicas foram associadas aos altos índices de exposição mecânica. O levantamento e manuseio de materiais resultaram em altas cargas nessas regiões do corpo, pode-se esperar que um alto índice de exposição física seja um dos fatores de risco.

No estudo de Rodrigues (2016) Foi identificada uma prevalência de sintomas osteomusculares entre os trabalhadores de 94,11\%, nos últimos 12 meses. Observa-se uma maior prevalência dos sintomas osteomusculares na região lombar (79,41\%), pescoço $(64,70 \%)$ e parte superior das costas $(58,82 \%)$. Do total, 30 participantes $(88,23 \%)$ afirmaram que percebem a relação dos sintomas relatados com o seu trabalho atual. Mais da metade da amostra apontou o seu trabalho como estressante $(52,94 \%)$ e cansativo $(52,94 \%)$. Os eixos temáticos mais abordados como causadores de dor foram o transporte de pacientes $(52,94 \%)$ seguido de posicionamento do paciente no leito $(38,23 \%)$. Verificou-se que os aspectos organizacionais do trabalho foram identificados por estes profissionais como contributivos para o possível adoecimento. Para os autores, a probabilidade de ter sintomas e eventualmente DORT aumenta quando os enfermeiros realizam trabalho por turnos longos, cuidados de higiene dos doentes e as ações relativas ao seu conforto na cama, sendo consideradas as tarefas com maior efeito na presença de sintomas de DORT na região lombar (Rodrigues, 2016).

A pesquisa de Carginin et al., (2019) com 301 profissionais da enfermagem de um Hospital Geral, para relacionar a dor lombar com suas cargas de trabalho, processos de desgaste e os riscos de adoecimento, mostrou que houve associação estatisticamente significativa entre as dimensões "organização do trabalho" e "condições de trabalho" com a dor lombar, que obtiveram classificação crítica, significando riscos moderados ao adoecimento profissional. Desta forma, a postura exercida durante o expediente pode ser influenciada pelo posto de trabalho e suas características como uma inadequada configuração arquitetônica dos serviços que limita o espaço de trabalho e o tipo de equipamentos utilizados, principalmente se não forem adequados às tarefas. Sempre que as condições referidas não estejam adaptadas ao trabalhador haverá tensão e compressão de músculos, exercendo maiores esforços mecânicos sobre o corpo, aumentando consideravelmente o risco para a ocorrência de DORT (Rodrigues, 2016; Carginin et al., 2019). 
Partindo desses pressupostos, é importante que os profissionais de saúde compreendam os aspectos que determinam o surgimento dos DORT, pois somente com essa compreensão pode-se perceber por que alguém pode apresentar dores, em alguns casos, sem a presença de lesões. Identificando os sintomas osteomusculares, é possível se realizar modificações que possam melhorar a organização, bem como a realização do trabalho dos profissionais de enfermagem, a fim de beneficiar esses trabalhadores e, por consequência, proporcionar melhorias no atendimento prestado (Ferreira, 2016; Arvidsson et al., 2016; Chiwaridzo et al., 2018; Elsherbeny et al., 2018; Brien et al., 2018).

No decorrer da pesquisa constatamos que, nos estudos nacionais e internacionais, as principais queixas e sintomatologias indicativas de DORT envolvem, principalmente, quadros álgicos em região lombar, cervical, membros superiores e inferiores, sendo a lombalgia o principal sintoma auto referido pelos profissionais da enfermagem. Os principais fatores etiológicos dos DORTS observados na presente revisão relacionam-se às posturas exercidas durante o labor, às longas jornadas de trabalho e aos altos índices de exposição mecânica, além do levantamento e manuseio de materiais, que resultam em altas cargas nas regiões do corpo mais relatadas com quadros álgicos (Ferreira, 2016; Rodrigues, 2016; Arvidsson et al., 2016; Freire et al., 2017; Chiwaridzo et al., 2018; Pereira, et al., 2018; Brien et al., 2018; Cargnin et al., 2019; Mazalo et al., 2021).

A maior prevalência dos sintomas na região lombar, deve-se a importância dessa região, para o trabalho dos profissionais de enfermagem, por ser uma região de sustentação do corpo, hipermóvel e de rotação do tronco e, muitas vezes, utilizada de forma inadequada pela má postura e por períodos prolongados. Notou-se uma forte relação da ocorrência de DORTS com o tempo de trabalho e tempo de exposição, relacionado a dinâmica de trabalho desses profissionais, significativamente associado ao desenvolvimento dos sintomas álgicos (Ferreira, 2016; Rodrigues, 2016; Arvidsson et al., 2016; Freire et al., 2017; Chiwaridzo et al., 2018; Pereira, et al., 2018; Brien et al., 2018; Cargnin et al., 2019; Mazalo et al., 2021).

\section{Conclusão}

A partir dos resultados obtidos na presente pesquisa, observou-se que existe um elevado índice de dores relacionadas ao trabalho nos profissionais enfermagem, visto que em todas as pesquisas analisadas, os participantes queixaram-se de quadros álgicos com prevalência na região lombar, associadas à organização do trabalho e à biomecânica exercida durante as atividades laborais, visto que em todas as pesquisas analisadas, os participantes queixaram-se de quadros álgicos associados às suas condições laborativas . Assim, percebe-se que há uma relação entre as posturas adotadas e as atividades executadas no ambiente de trabalho com o desenvolvimento de distúrbios osteomusculares que causam dor. Ademais, nota-se que os sintomas precisam ser identificados precocemente, evitando que as dores se agravem e que pequenos distúrbios virem lesões.

O estudo contribuiu para mostrar a ocorrência de DORT como uma problemática de saúde desses profissionais e a necessidade de seu monitoramento e vigilância. Seus resultados demonstram a importância de se investir no cenário laboral da prática da enfermagem e melhorar suas condições ergonômicas.

Sugere-se a realização de estudos mais aprofundados acerca da temática, que possam embasar intervenções cada vez mais assertivas em função da qualidade de vida no trabalho e, principalmente, da saúde desses profissionais.

\section{Referências}

Almeida, A. M. D. O., Lima, A. K. G., Vasconcelos, M. G. F., Lima, A. C. S., \& Oliveira, G. Y. M. D. (2016). Estresse ocupacional em enfermeiros que atuam em cuidados ao paciente crítico. Rev. enferm. UFPE on line, 1663-1671.

Anap, D. B., Iyer, C., \& Rao, K. (2013). Work related musculoskeletal disorders among hospital nurses in rural Maharashtra, India: a multi centre survey. Int J Res Med Sci, 1(2), 101-7.

Arvidsson, I., Simonsen, J. G., Dahlqvist, C., Axmon, A., Karlson^, B., Björk, J., \& Nordander, C. (2016). Cross-sectional associations between occupational factors and musculoskeletal pain in women teachers, nurses and sonographers. BMC musculoskeletal disorders, 17(1), 1-15. 
Bezerra, S. K. F., \& Costa, E. C. (2016). Ginástica laboral como proposta de intervenção e promoção da saúde dos profissionais de enfermagem.

Brien, K., Lukhele, Z., Nhlapo, J. M., Pieterse, A., Swanepoel, A., Wagener, L., \& Mashola, M. K. (2018). Work-related musculoskeletal disorders in nurses working in South African spinal cord rehabilitation units.

Cargnin, Z. A., Schneider, D. G., Vargas, M. A. D. O., \& Machado, R. R. (2019). Non-specific low back pain and its relation to the nursing work process. Revista latino-americana de enfermagem, 27.

Chiwaridzo, M., Makotore, V., Dambi, J. M., Munambah, N., \& Mhlanga, M. (2018). Work-related musculoskeletal disorders among registered general nurses: a case of a large central hospital in Harare, Zimbabwe. BMC research notes, 11(1), 1-7.

Chung, Y. C., Hung, C. T., Li, S. F., Lee, H. M., Wang, S. G., Chang, S. C. \& Yang, J. H. (2013). Risk of musculoskeletal disorder among Taiwanese nurses cohort: a nationwide population-based study. BMC musculoskeletal disorders, 14(1), 1-6.

Cilliers, L., \& Maart, S. (2013). Attitudes, knowledge and treatment of low back pain amongst nurses in the Eastern Cape, South Africa. African journal of primary health care \& family medicine, 5(1).

Costa, A. J. F. \& Dias, B. L (2015). Importância de um programa de exercícios na prevenção de LER/DORT. UNILUS Ensino e Pesquisa, $13(30), 223$.

Elsherbeny E. E., et al. (2018). Prevalence and associated factors of musculoskeletal complaints among nurses of mansoura university children hospital. Egyptian Journal of Occupational Medicine, 42(2), 151-166.

Estrela, C. (2018). Metodologia científica: ciência, ensino, pesquisa. Artes Médicas.

Ferri, J. F. A. \& Xavier, D. P. (2015). Qualidade de vida no trabalho dos profissionais de enfermagem em ambiente Hospitalar: uma revisão integrativa. Revista Espaç. Saúde, 16(1): 66-74.

Fonseca, R., \& Serranheira, F. (2006). Sintomatologia musculoesquelética auto-referida por enfermeiros em meio hospitalar. Revista Portuguesa de saúde pública, 6(Temático-Saúde ocupacional), 37-44.

França, F. M. D., Ferrari, R., Ferrari, D. C., \& Alves, E. D. (2012). Burnout e os aspectos laborais na equipe de enfermagem de dois hospitais de médio porte. Revista Latino-Americana de Enfermagem, 20, 961-970.

Freire, L. A., Soares, T. C. N., \& dos Santos Torres, V. P. (2017). Influência da ergonomia na biomecânica de profissionais de enfermagem no ambiente hospitalar. Biológicas \& Saúde, 7(24).

FREITAS, C. (2012). Reabilitação dinâmico-funcional da coluna lombar. São Paulo: Phorte, 29-39.

Jellad, A., Lajili, H., Boudokhane, S., Migaou, H., Maatallah, S., \& Frih, Z. B. S. (2013). Musculoskeletal disorders among Tunisian hospital staff: Prevalence and risk factors. The Egyptian Rheumatologist, 35(2), 59-63.

Koche, J. C. (2011). Fundamentos de Metodologia Científica: teoria da ciência e iniciação à pesquisa. Petrópolis. Rio de Janeiro. Vozes. 185p.

Lelis, C. M., Battaus, M. R. B., Freitas, F. C. T. D., Rocha, F. L. R., Marziale, M. H. P., \& Robazzi, M. L. D. C. C. (2012). Work-related musculoskeletal disorders in nursing professionals: an integrative literature review. Acta Paulista de Enfermagem, 25, 477-482..

Luan, H. D., Hai, N. T., Xanh, P. T., Giang, H. T., Van Thuc, P., Hong, N. M., \& Khue, P. M. (2018). Musculoskeletal disorders: prevalence and associated factors among district hospital nurses in Haiphong, Vietnam. BioMed research international, 2018.

Lucca, S. R. (2017). Saúde, Saúde Mental, Trabalho e Subjetividade. Revista Laborativa, 6(1 esp), 147-159.

Lucca, S. R. D., \& Rodrigues, M. S. D. (2015). Absenteísmo dos profissionais de enfermagem de um hospital universitário do estado de São Paulo, Brasil. Rev Bras Med Trab, 13(2), 76-82.

Martins, E. A., Correa, C. S., \& Vidal, P. C. V. (2017). Doenças osteomusculares relacionadas ao trabalho com destaque aos profissionais da área de enfermagem. Revista Perspectiva: Ciência e Saúde, 2(2).

Mazalo, J. V., Mori, B., De Paulo, T. R., Pinheiro, Q. N., \& Boechat, A. L. (2021). Distúrbios osteomusculares relacionados ao trabalho em enfermeiros de um hospital público em Manaus-AM. Desafios-Revista Interdisciplinar da Universidade Federal do Tocantins, 8(2), 56-65.

Neves, M., \& Serranheira, F. (2014). A formação de profissionais de saúde para a prevenção de lesões musculoesqueléticas ligadas ao trabalho a nível da coluna lombar: uma revisão sistemática. Revista Portuguesa de Saúde Pública, 32(1), 89-105.

Paula, E. A. D., \& Amaral, R. M. M. F. D. (2019). Atuação interdisciplinar em grupos de qualidade de vida para pacientes com Lesões por esforços repetitivos/Distúrbios osteomusculares relacionados ao trabalho-LER/DORT. Revista Brasileira de Saúde Ocupacional, 44.

Pinhatti, E. D. G., Ribeiro, R. P., Soares, M. H., Martins, J. T., Lacerda, M. R., \& Galdino, M. J. Q. (2018). Psychosocial aspects of work and minor psychic disorders in nursing: use of combined models. Revista latino-americana de enfermagem, 26.

Punnett, L., \& Wegman, D. H. (2004). Work-related musculoskeletal disorders: the epidemiologic evidence and the debate. Journal of electromyography and kinesiology, 14(1), 13-23.

Reis, P. F. (2016). O gênero e sua influênia na prevalência de distúrbios musculoesqueléticos em enfermeiros. Blucher Engineering Proceedings, 3(3), 943-952.

Ribeiro, T. et al. (2017). Work related musculoskeletal disorders in primary health care nurses. Applied Nursing Research, 3: 72-77. 
Research, Society and Development, v. 10, n. 15, e149101523031, 2021

(CC BY 4.0) | ISSN 2525-3409 | DOI: http://dx.doi.org/10.33448/rsd-v10i15.23031

Rodrigues, C. M. D. A. (2016). Sintomas osteomusculares relacionados ao trabalho de enfermagem em uma unidade de terapia intensiva: uma abordagem sobre LER/DORT (Master's thesis).

Santos, R. P., \& Andrade, E. N. (2011). Frequência e fatores associados dos sintomas musculoesqueléticos em auxiliares de enfermagem. Fisioter. Bras, 12(1):20-24.

Shieh, S. H., Sung, F. C., Su, C. H., Tsai, Y., \& Hsieh, V. C. R. (2016). Increased low back pain risk in nurses with high workload for patient care: A questionnaire survey. Taiwanese Journal of Obstetrics and Gynecology, 55(4), 525-529.

Sorour, A. S., \& El-Maksoud, M. M. (2012). Relationship between musculoskeletal disorders, job demands, and burnout among emergency nurses. Advanced emergency nursing journal, 34(3), 272-282.

Sousa, M. N. A., Da Silva, G. M., Costa, T. S., Nunes, R. M. V., \& Medeiros, H. R. L. (2015). Prevalência de distúrbios osteomusculares em enfermeiros. FIEP Bulletin - online, 85(1): 775-780.

Vieira, M. V. P., \& de Alcântara, D. S. (2013). Prevalência de dor lombar crônica em trabalhadores de enfermagem: revisão bibliográfica. Amazônia: Science \& Health, 1(3), 49-55. 